\title{
Primary care of mothers and infants by the same or different physicians: a population-based cohort study
}

\author{
Natasha R. Saunders MD MSc, Joel G. Ray MD MSc, Christina Diong MSc, Jun Guan MSc, Eyal Cohen MD MSc
}

Cite as: CMAJ 2020 September 8;192:E1026-36. doi: 10.1503/cmaj.191038

\begin{abstract}
BACKGROUND: Different primary care delivery models exist for mothers and their infants. We examined whether primary care system performance measures differed when mother-infant dyads received primary care from the same or different providers.
\end{abstract}

METHODS: We conducted a populationbased cohort study using Ontario health administrative data from 2004 to 2016. We included primiparous women and their singleton term infants and classified the primary care practitioners who provided the majority of care to the infant and the mother as concordant (same family physician for both; reference group), discordant (a different family physician for each) or pediatrician (pediatrician for the child, family physician for the mother). The primary outcome was nonobstetric maternal hospital admissions between 42 days and 2 years after delivery.

RESULTS: Among 481721 mother-child pairs, $239033(49.6 \%)$ received concordant care, $114006(23.7 \%)$ received discordant care, and 128682 (26.7\%) received pediatrician care. Mothers in the pediatrician group were older and had greater comorbidity. Relative to concordant care, maternal nonobstetric hospital admissions occurred similarly under discordant care (adjusted odds ratio [OR] 1.00, 95\% confidence interval [Cl] 0.96-1.04) and in the pediatrician group (adjusted OR 0.99, 95\% Cl 0.951.02). Maternal deaths were similar under discordant care (adjusted OR $1.00,95 \% \mathrm{Cl} 0.62-1.63$ ) but lower in the pediatrician group (adjusted OR 0.55 ,
95\% Cl 0.34-0.89). Maternal primary care visits were lower in both the discordant group (adjusted relative risk [RR] $0.68,95 \% \mathrm{Cl} 0.68-0.69$ ) and the pediatrician group (adjusted RR $0.75,95 \% \mathrm{Cl}$ $0.75-0.76$ ). Healthy children were more likely to miss the enhanced 18-month well-baby visit under discordant care (adjusted OR 1.06, 95\% Cl 1.03-1.09) but less likely to miss this visit under pediatrician care (adjusted OR $0.47,95 \% \mathrm{Cl}$ 0.46-0.49).

INTERPRETATION: Concordant care provided to a new mother and her infant by the same family physician was not associated with better primary care health system performance. The reason that pediatric primary care is associated with better maternal and child outcomes remains to be determined.
A round the time of birth, parents of healthy newborns must begin the search for a primary care physician for their child. According to published clinical guidelines, 10 well-child visits are recommended in the first 2 years of life. ${ }^{1,2}$ The demands of prioritizing the care of her young child may distract a new mother from accessing primary care for herself, or maintaining continuity of care. Hence, a family physician who cares for both a mother and her infant may conceivably facilitate integrated and coordinated primary care delivery for each patient. Conversely, such an approach might lead to distracted care, there being a preoccupation with the child's needs over those of the mother.

Across high-income countries, wide variation exists in primary care delivery models for young families..$^{3-5}$ In some jurisdictions - the United Kingdom, Australia and Denmark - care is provided through a family physician-only model. In others, including the United States, Israel and Spain, primary child care is provided by a pediatrician and primary adult care by a family physician. Other countries, including France, Germany and Canada, offer both these options., Which approach is best has not been formally assessed using existing primary care health system performance measures, such as unplanned hospital admissions, emergency department visits, or maternal and child death.

In some provinces and regions of Canada, women have a choice about their primary care provider. Within Ontario, Canada's most populous province, several models of primary care are available. These include capitated remuneration models (in which physicians receive a set amount of funding per patient in their practice), noncapitated models (in which physicians are 
compensated primarily through fee-for-service but are also eligible for specific bonuses and premiums based on patient enrolment or provision of after-hours services) and fee-forservice models (in which physicians bill and are remunerated according to each patient visit or service provided). Ontario funds health care for eligible residents, and linked health administrative data sets permit the collection of population-based performance indicators of quality primary care, including unplanned hospital admissions, emergency department use, rates of preventable illness (including cardiovascular disease) and all-cause mortality. ${ }^{7-15}$ For young children, well-baby screening is within the list of quality indicators. ${ }^{13}$

We examined whether primary care health system performance measures differed when a mother and infant received primary care from the same family physician, from separate family physicians, or from a pediatrician for the child and a family physician for the mother. We hypothesized that concordant care by the same family physician would result in improved outcomes for the woman, without compromising the outcomes of her young child in the first 2 years of life.

\section{Methods}

\section{Setting}

We conducted a population-based cohort study in Ontario, Canada, using linked health and administrative data for women and their singleton infants who were eligible for provincial health insurance and who were admitted to hospital for delivery of a live-born infant between 2005 and 2014.

\section{Study population}

We included primiparous mothers and their children born between Apr. 1, 2005, and Mar. 31, 2014. We limited the cohort to primiparous births to focus on new mothers navigating the child health care system for the first time. We excluded mothers who became Ontario residents less than 365 days before the birth date of their infant, mothers younger than 18 years or older than 55 years at the child's birth date, and those with multifetal births. We also excluded mother-infant dyads in which the mother or child died less than 42 days after the birth date and those for whom the hospital discharge date of the mother or the child was more than 42 days after the child's birth date (Appendix 1, Figure A1-1, available at www.cmaj.ca/lookup/suppl/doi:10.1503/ cmaj.191038/-/DC1).

\section{Exposure groups}

We identified primary care practitioners who provided the majority of primary care to infants and their mothers between 42 and 365 days of life, using outpatient physician billing codes for primary care visits (Appendix 1, Box A1-1). This majority care designation was assigned to the physician who provided the greatest number of days of visits for primary care during the observation window. We classified primary care as follows: concordant care, where both the mother and the infant had the same family physician; discordant care, where the mother and infant had different family physicians providing most primary care (i.e., 2 separate family physicians); and pediatrician care, where a pediatrician provided primary care to the infant and a family physician provided care to the mother. We excluded dyads in which either the mother or the infant had no primary care visits, as well as those in which the mother received the majority of primary care from a pediatrician or other specialist (e.g., obstetrician). We assigned mothers or their infants who received the majority of primary care through community health centres (reflecting $<1 \%$ of the population) or from a nurse practitioner (where physician billing records are not always captured) to the category of the provider they visited the second highest number of times.

As a sensitivity analysis, we used a more sensitive, and less specific, definition of concordance by classifying as "concordant care" mother-child dyads with any visits (rather than the majority of visits) to the same provider for primary care during the 42to 365-day observation window (see Appendix 1, Box A1-1, for codes describing primary care assignment for analyses).

\section{Study outcomes}

The Ontario Primary Care Performance Measurement Framework outlines several measures for evaluating primary care at the system and practice levels using multiple methods of data collection. ${ }^{15}$ While the study outcomes were not all-inclusive, we chose outcomes for the current study that could be mapped onto this existing framework, ascertained using health and administrative data. The primary study outcome was any maternal nonobstetric hospital admission between 42 days (corresponding to the end of the postpartum period) and 2 years after the child's birth date. In Ontario, hospital admissions for conditions managed in primary care have been shown to be related to primary care remuneration models and are a measure of the integration and effectiveness of primary care provision..$^{15,16}$

We also analyzed the following secondary outcomes: maternal and child deaths; all-cause and low-acuity emergency department visits (each of which are measures of access and integration); primary care visits in the 2 years after the birth date (a measure of system and practice efficiency); maternal cardiovascular disease (a measure of care effectiveness), defined as a composite of any in-hospital diagnosis of coronary artery disease, cerebrovascular disease, peripheral artery disease, cardiac dysrhythmia or heart failure; maternal mental health hospital admissions (a measure of health outcome); hospital admissions for the child; and nonreceipt of an enhanced 18-month well-baby visit (a measure of population health preventive care), defined as a publicly funded visit to a health care provider in which standardized developmental review and evaluation tools for toddlers are paired with a routine well-baby check-up. ${ }^{13}$ The specific data sources and codes used to define each outcome are presented in Appendix 1 (Box A1-1, Box A1-2 and Table A1-1). We performed another sensitivity analysis using a narrower, more proximal outcome window of 42 to 365 days after the birth date for nonmaternity hospital admissions.

\section{Data sources}

We used several linked health and demographic data sets available at ICES, a not-for-profit research institute whose legal status 
under Ontario's health information privacy law allows it to collect and analyze health data without consent. We linked individual patient-level records in the data sets using a unique encoded identifier derived from the health card number of every resident in Ontario insured with provincial health insurance.

We identified in-hospital obstetric deliveries using the ICES MOMBABY data set. All in-hospital deliveries in Ontario are included in this validated data set, with $98 \%$ deterministic linkage of newborn and maternal hospital records. ${ }^{17}$ Data for fathers and adopted newborns were not available from the existing data sets. We obtained demographic information from Ontario's Health Insurance Registry (Registered Persons Database), including the child's date of birth and the postal code of both the mother and the infant. We linked postal codes to Canadian census data to obtain neighbourhood-level income and to determine rural or urban residence. We obtained the mother's immigration status from the Permanent Resident Database of Immigration, Refugees and Citizenship Canada. ${ }^{18}$ We obtained the practice and remuneration model types of primary care providers from the Corporate Provider Database. We obtained clinical information about all hospital discharges from the Canadian Institute for Health Information Discharge Abstract Database, which uses standard diagnostic codes from the International Statistical Classification of Diseases and Related Health Problems, 10th revision, Canadian version (ICD10-CA) and procedural or interventional codes from the Canadian Classification of Health Interventions. We identified emergency department records using the National Ambulatory Care Reporting System, which includes all emergency department, day surgery and hospital outpatient clinic visits. We used the Ontario Health Insurance Plan physician billings database to obtain information about outpatient physician and visit characteristics. The Ontario Mental Health Reporting System contains mental health clinical and administrative data for adult patients in designated inpatient mental health beds in the province; we used this source to ascertain mental health-related hospital admissions.

ICES data are valid for sociodemographic characteristics, physician billing claims and primary hospital diagnoses. ${ }^{19}$

\section{Statistical analysis}

For the baseline characteristics of the mothers and their newborns, we calculated means, medians or rates.

We used multivariable logistic regression to generate odds ratios (ORs) and 95\% confidence intervals (Cls) for the relation between primary care provision type and the outcomes of maternal hospital admission, child hospital admission, maternal death, child death, the composite outcome for cardiovascular disease (limited to the mothers) and receipt of the enhanced 18-month well-baby visit (limited to the children). The ORs were adjusted for maternal age, neighbourhood income quintile, rurality, immigration status, number of medical comorbidities, family physician practice and remuneration model, ${ }^{20}$ the child's gestational age at birth, the child's birth weight, the child's complex chronic conditions and the child's length of stay for the birth hospitalization, given the biologically and clinically plausible association of each factor with each outcome. Because a child with a complex chronic condition is more likely to be cared for by a pediatrician, we re-ran the above models with stratification by the child's general health, as term healthy children and children with a complex chronic condition or preterm birth.

Separately, for the outcomes of the number of primary care visits for the mother and the number of such visits for the child, we used negative binomial regression, with adjustment for the aforementioned covariables, to generate relative risks (RRs) comparing the rates of these events occurring. For the outcomes of mother and child all-cause emergency department visits, as well as low-acuity emergency department visits, we used zeroinflated negative binomial regression to generate RRs, again using the same covariables.

For maternal and child hospital admissions, we described the most responsible diagnosis from the discharge record for the 15 most common diagnoses, using ICD-10-CA codes (Appendix 1, Table A1-2).

To assess the construct validity of the assignment of primary care providers in this study, we further described the proportions of mother-child dyads with high continuity of care $(\geq 76 \%$ of all primary care visits to their assigned provider) and those with lower continuity of care $(<76 \%$ of all primary care visits to their assigned provider). We also described the proportion of children who received an enhanced 18-month well-baby visit by their assigned provider (Appendix 1, Table A1-3).

We used SAS Enterprise Guide, version 6.1 (SAS Institute) for all statistical analyses.

\section{Ethics approval}

The Research Ethics Board at The Hospital for Sick Children, Toronto, approved this study.

\section{Results}

After exclusions, we included a total of 481721 mother-child dyads in the study (Appendix 1, Figure A1-1). Of these, 239033 $(49.6 \%)$ received concordant care, 114006 (23.7\%) received discordant care, and $128682(26.7 \%)$ received pediatrician care.

Mothers in the pediatrician group were older on average (30.4 yr) than those in the discordant (28.4 yr) or concordant (28.8 $\mathrm{yr}$ ) family physician groups, were most likely to reside in the highest neighbourhood income quintile $(17.8 \%, 15.8 \%$ and $15.7 \%$, respectively), and had the highest proportion of immigrant mothers (37.5\%, $19.3 \%$ and $22.4 \%$, respectively) (Table 1 ). Mothers in the discordant family physician group less frequently had more than 10 comorbidities (16.2\%), in contrast to those in the concordant $(24.7 \%)$ and pediatrician $(25.5 \%)$ groups.

In the concordant family physician group, the physician of the mother most often practised in noncapitated remuneration models $(52.0 \%)$, followed by capitated models (37.1\%). Physicians of mothers in the pediatrician model most often practised in fee-for-service remuneration models $(50.3 \%)$ followed by noncapitated models (36.9\%). The majority of providers caring for mothers in the discordant family physician group practised in a fee-for-service remuneration model (80.9\%). 
Table 1: Characteristics of the study cohort according to the primary care provider of the mother and her child

Group;* no. (\%) of mother-child pairs $\dagger$

\begin{tabular}{|c|c|c|c|}
\hline Characteristic & $\begin{array}{l}\text { Concordant } \\
n=239033\end{array}$ & $\begin{array}{l}\text { Discordant } \\
n=114006\end{array}$ & $\begin{array}{c}\text { Pediatrician } \\
n=128682\end{array}$ \\
\hline \multicolumn{4}{|l|}{ Mother } \\
\hline Age, mean $\pm S D$, yr & $28.8 \pm 5.2$ & $28.4 \pm 5.3$ & $30.4 \pm 5.2$ \\
\hline \multicolumn{4}{|c|}{ Neighbourhood income quintile } \\
\hline Q1 (lowest) & $48881(20.4)$ & $24415(21.4)$ & 26849 (20.9) \\
\hline Q2 & $49335(20.6)$ & $23473(20.6)$ & $26105(20.3)$ \\
\hline Q3 & $51562(21.6)$ & $23820(20.9)$ & $24570(19.1)$ \\
\hline Q4 & $51086(21.4)$ & $23728(20.8)$ & $27748(21.6)$ \\
\hline Q5 (highest) & $37478(15.7)$ & $18031(15.8)$ & $22909(17.8)$ \\
\hline Missing & $691(0.3)$ & $539(0.5)$ & $501(0.4)$ \\
\hline Rural residence & $15952(6.7)$ & $10066(8.8)$ & $1009(0.8)$ \\
\hline \multicolumn{4}{|l|}{ Immigration status } \\
\hline Nonimmigrant & $185472(77.6)$ & $91985(80.7)$ & $80373(62.5)$ \\
\hline Nonrefugee immigrant & 47595 (19.9) & $19346(17.0)$ & $42700(33.2)$ \\
\hline Refugee immigrant & $5966(2.5)$ & $2675(2.3)$ & $5609(4.4)$ \\
\hline \multicolumn{4}{|c|}{ Comorbidities in preceding $3 \mathrm{yr} \ddagger$} \\
\hline $0-5$ & $65718(27.5)$ & $44715(39.2)$ & $35418(27.5)$ \\
\hline $6-9$ & $114313(47.8)$ & $50837(44.6)$ & $60444(47.0)$ \\
\hline$\geq 10$ & $59002(24.7)$ & $18454(16.2)$ & $32820(25.5)$ \\
\hline \multicolumn{4}{|l|}{ Primary care model§ } \\
\hline Fee-for-service & $25848(10.8)$ & $92217(80.9)$ & $64730(50.3)$ \\
\hline Noncapitated & $124212(52.0)$ & $13132(11.5)$ & 47422 (36.9) \\
\hline Capitated & $88618(37.1)$ & $8578(7.5)$ & $16438(12.8)$ \\
\hline Other & $355(0.1)$ & $79(0.1)$ & $92(0.1)$ \\
\hline \multicolumn{4}{|l|}{ Child } \\
\hline \multicolumn{4}{|l|}{ Gestational age at birth, wk } \\
\hline$<34$ & $1803(0.8)$ & $1197(1.0)$ & $2199(1.7)$ \\
\hline $34-36$ & $11708(4.9)$ & $5355(4.7)$ & $7796(6.1)$ \\
\hline$\geq 37$ & $225455(94.3)$ & $107405(94.2)$ & $118659(92.2)$ \\
\hline Mean \pm SD & $39.1 \pm 1.6$ & $39.1 \pm 1.8$ & $38.9 \pm 1.8$ \\
\hline \multicolumn{4}{|l|}{ Birth weight, g } \\
\hline$<1500$ & $303(0.1)$ & $430(0.4)$ & $505(0.4)$ \\
\hline $1500-2500$ & $9989(4.2)$ & $4700(4.1)$ & $8195(6.4)$ \\
\hline $2501-4000$ & $204395(85.5)$ & $97084(85.2)$ & $109329(85.0)$ \\
\hline$>4000$ & $24315(10.2)$ & $11690(10.3)$ & $10636(8.3)$ \\
\hline Mean \pm SD & $3380 \pm 510$ & $3370 \pm 530$ & $3300 \pm 540$ \\
\hline \multicolumn{4}{|c|}{ Birth hospitalization length of stay } \\
\hline$\leq 24 \mathrm{~h}$ & $11715(4.9)$ & $6431(5.6)$ & $3942(3.1)$ \\
\hline $2-6 d$ & $219341(91.8)$ & $103473(90.8)$ & $117095(91.0)$ \\
\hline$\geq 7 d$ & $7977(3.3)$ & $4102(3.6)$ & $7645(5.9)$ \\
\hline Mean $\pm S D, h$ & $63.5 \pm 72.0$ & $63.4 \pm 75.1$ & $75.8 \pm 106.5$ \\
\hline Complex chronic condition & $8802(3.7)$ & $4693(4.1)$ & $8006(6.2)$ \\
\hline
\end{tabular}

Note: $\mathrm{SD}=$ standard deviation.

*Concordant model means mother and child received care from the same family physician; discordant model means the mother and child received care from different familiy physicians; the pediatrician model means the mother received care from a family physician and the child from a pediatrician.

†Except where indicated otherwise.

$\ddagger$ According to Johns Hopkins Adjusted Diagnostic Groups.

$\S$ In Ontario, several models of primary care practice and remuneration exist. A full description of the models is available elsewhere..$^{20}$ Broadly, fee-for-service is a traditional model in which physicians are paid for each service provided and typically work in solo practice, with no requirements for after-hours care. In noncapitated models, physicians are paid largely through fee-for-service, but they receive incentives and bonuses and a small fee for rostering patients; practice is often in a group, with after-hours premiums. Capitated models involve payment for each rostered patient blended with fee-for-service and incentive and bonus components; practice is in a group, with after-hours premiums. 
More infants in the pediatrician model were born preterm, were small for gestational age, had a prolonged hospital stay at birth and had a complex chronic condition (Table 1). These patterns among the groups were similarly seen when we used a more sensitive definition of the primary care provider (Appendix 1, Table A1-4).

\section{Maternal outcomes}

The primary study outcome of maternal nonobstetric hospital admission did not differ between women in the concordant care group and those in the discordant care group (adjusted OR 1.00, $95 \% \mathrm{Cl} 0.96-1.04$ ) or in the pediatrician group (adjusted OR 0.99, 95\% Cl 0.95-1.02) (Table 2, Figure 1 and Appendix 1, Figure A1-2A). However, when we used the more sensitive definition of primary care concordance, the odds of hospital admission were marginally lower under both discordant care (adjusted OR 0.95, 95\% Cl 0.911.00) and pediatrician care (adjusted $0.96,95 \% \mathrm{Cl} 0.92-0.99$ ) (Appendix 1, Table A1-5); similarly, when we used a more proximal and shorter observation window after the child's birth date, the odds of admission were marginally lower both for discordant care (adjusted OR 0.90, 95\% Cl 0.83-0.97) and for pediatrician care (adjusted OR 0.93, 95\% Cl 0.87-0.99) (Appendix 1, Table A1-6). Decreased odds of maternal nonobstetric hospital admission were also independently associated with age (per additional year, adjusted OR 0.96, 95\% $\mathrm{Cl} 0.95-0.96$ ), urban residence (adjusted OR $0.68,95 \% \mathrm{Cl} 0.65-0.72$ ) and immigration status (nonrefugees, adjusted OR $0.75,95 \% \mathrm{Cl} 0.72-0.78$; refugees, adjusted OR 0.90, 95\% Cl 0.83-0.98).

Compared with a fee-for-service maternal primary care provider remuneration and practice model, noncapitated models (adjusted OR 1.17, 95\% Cl 1.13-1.22) and capitated models (adjusted OR 1.17, 95\% Cl 1.12-1.22) were independently associated with nonobstetric hospital admissions (Table 2 and Appendix 1, Table A1-7). Increased odds of maternal nonobstetric hospital admissions were also associated with previous mental health admissions (adjusted OR 1.56, 95\% Cl 1.40-1.73) and greater comorbidity ( $\geq 10$ v. $0-5$ comorbidities, adjusted OR 1.53 , 95\% Cl 1.47-1.59; 6-9 v. 0-5 comorbidities, adjusted OR 1.13, 95\% Cl 1.09-1.17) (Table 2).

The odds of cardiovascular outcomes did not differ between primary care groups (Figure 1), but maternal death was lower in the pediatrician group compared with concordant care (adjusted OR 0.55, 95\% Cl 0.34-0.89) (Figure 1 and Appendix 1, Figure A1-2B and Table A1-5). Hospital admission for mental illness was less likely in the pediatrician group than under concordant care (adjusted OR $0.86,95 \% \mathrm{Cl} 0.76-0.99$ ) but was not significantly different under discordant care (adjusted OR 1.12, 95\% Cl 0.98-1.28) (Figure 1). Relative to the concordant care group, maternal primary care visits in the first 2 years were lower under discordant care (adjusted RR 0.68, 95\% Cl 0.680.69 ) and pediatrician care (adjusted RR $0.75,95 \% \mathrm{Cl} 0.75-0.76$ ) (Figure 2), whereas maternal emergency department visits were lowest under pediatrician care (adjusted RR 0.87, 95\% Cl 0.850.88 ) and slightly but significantly higher under discordant care (adjusted RR 1.06, 95\% Cl 1.04-1.08) (Figure 2). These findings were robust under the specific and sensitive definitions of pri- mary care concordance for most outcomes (Appendix 1, Table A1-5 and Table A1-8).

\section{Child outcomes}

Compared with concordant care, hospital admission of healthy children in the first 2 years of life was less common under discordant care (adjusted OR 0.92, 95\% Cl 0.89-0.96) and more likely under pediatrician care (adjusted OR 1.14, 95\% Cl 1.101.18), with similar but slightly stronger associations among children with complex chronic conditions (Figure 3 and Appendix 1, Figure A1-2C and Table A1-9). Child deaths were rare, but among children with chronic conditions, deaths were higher under discordant care (adjusted OR 1.80, 95\% Cl 1.142.83) and pediatrician care (adjusted OR 1.72 , 95\% Cl 1.142.60). A similar association for child death was seen among healthy children with discordant care (adjusted OR 1.98, 95\% $\mathrm{Cl}$ 1.24-3.15), but the association was not significant for pediatrician care (adjusted OR 1.45, 95\% Cl 0.92-2.28) (Figure 3 and Appendix 1, Figure A1-2D). Relative to concordant care, children under discordant care were more likely to miss the enhanced 18-month well-baby visit (adjusted OR 1.31, 95\% Cl 1.28-1.34), although this was less likely under pediatrician care (adjusted OR $0.53,95 \% \mathrm{Cl} 0.52-0.54$ ). Primary care visits in the first 2 years of life were lower under pediatrician care (adjusted RR 0.93, 95\% Cl 0.93-0.94) and discordant care (adjusted RR 0.80, 95\% Cl 0.79-0.80). All-cause (adjusted OR $0.85,95 \% \mathrm{Cl} 0.85-0.86$ ) and low-acuity (adjusted OR 0.65, 95\% $\mathrm{Cl}$ 0.64-0.66) emergency department visits were lowest under pediatrician care (Figure 2).

Sensitivity analyses using a more inclusive definition of concordance showed similar patterns of child health and health access outcomes (Appendix 1, Tables A1-8, A1-9 and A1-10).

\section{Interpretation}

In this large population-based cohort study of primiparous mothers and their young children, concordant care by the same family physician was not associated with improved maternal health or health system performance measurement outcomes. However, primary care provided by a pediatrician was associated with some better maternal outcomes and lower health system utilization in the first 2 years after giving birth, even after accounting for comorbidities and sociodemographic differences. Primary care by a pediatrician was also associated with greater child health system performance metrics, including enhanced primary care visits and fewer emergency department visits.

We originally hypothesized that there would be improved outcomes among mothers receiving concordant primary care. Instead, we found that outcomes either did not differ or were sometimes better in mothers with a dedicated family physician whose child received care from a pediatrician, and were either no different or marginally worse in mother-child dyads with 2 distinct family physicians. Although these observations are not causal, and the associations were often modest, our results were unexpected, and we considered several explanations. First, 
Table 2: Number, proportion, and unadjusted and adjusted odds ratios for maternal nonobstetric hospital admissions, according to the primary care provider of the mother and of her infant and by their characteristics

OR $(95 \% \mathrm{CI})$

\section{Characteristic}

\section{Primary care concordance group}

Overall

Concordant (1 family physician)

Discordant (2 family physicians)

Pediatrician (pediatrician + family physician)

\section{Characteristics of the mother}

Primary care model

Fee-for-service

Noncapitated

Capitated

Other

Age, per additional yr

Neighbourhood income quintile

Q1 (lowest)

Q2

Q3

Q4

Q5 (highest)

Residence

Rural

Urban

Immigration status

Nonimmigrant

Nonrefugee immigrant

Refugee immigrant

Comorbidities in preceding $3 \mathrm{yr}^{\star}$

$0-5$

6-9

$\geq 10$

Previous mental health hospitalization

No

Yes

\section{Characteristics of the child}

Gestational age at birth, per additional week

Birth weight, per additional $1000 \mathrm{~g}$

Birth hospitalization length of stay, per additional day

Complex chronic condition

No

Yes
No. of dyads

481721

239033

114006

128682

182795

184766

113634

526

$-$

100145

98913

99952

102562

78418

27027

454694

357830

109641

14250

145851

225594

110276

477969

3752

22180 (4.64)

395 (10.53)

$18077(5.05)$
$3844(3.51)$
$654(4.59)$

5685 (3.90)

10072 (4.46)

$6818(6.18)$

5160 (5.15)

4624 (4.67)

4686 (4.69)

4533 (4.42)

3464 (4.42)

$1984(7.34)$

$20591(4.53)$

1.00 (ref.)

$0.60(0.57-0.63)$

1.00 (ref.)

$0.68(0.66-0.71)$

$0.90(0.83-0.98)$

1.00 (ref.)

1.15 (1.11-1.19)

1.62 (1.57-1.68)

1.00 (ref.)

$2.42(2.18-2.69)$

$0.95(0.95-0.96)$

$0.93(0.90-0.95)$

$1.00(1.00-1.01)$

21500 (4.67)

460220

21501

\section{$21500(4.67)$}

$1075(5.00)$
Adjusted

Note: $\mathrm{Cl}=$ confidence interval, $\mathrm{OR}=$ odds ratio, ref. = reference category *According to Johns Hopkins Adjusted Diagnostic Groups. 


\section{Outcomes for mothers \\ Hospital admission \\ Concordant \\ Discordant \\ Pediatrician}

Death

Concordant

Discordant

Pediatrician

$73 / 239033(0.031)$

42/114 006 (0.037)

$27 / 128682(0.021)$

\section{Cardiovascular outcome}

Concordant

109/239033 (0.046)
Adjusted OR

$(95 \% \mathrm{CI})$

5192/114 $006(4.6) \quad 1.00(0.96-1.04)$

$5338 / 128682(4.1) \quad 0.99(0.95-1.02)$

$1.00(0.62-1.63)$

$0.55(0.34-0.89)$

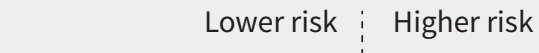

Discordant

Pediatrician

$37 / 114006(0.032)$

$43 / 128682(0.033)$

\section{Hospital admission for mental health care}

Concordant

Discordant

Pediatrician
971/239 $033(0.4)$

$538 / 114006(0.5)$

$392 / 128682(0.3)$

(1)

1.00 (ref.)

$1.12(0.98-1.28)$

$0.86(0.76-0.99)$

$0.94(0.59-1.48)$

$0.71(0.49-1.05)$

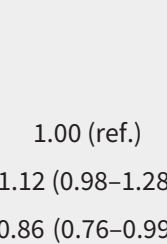

\section{ED visit for mental health care}

Concordant

$3487 / 239033$ (1.5)

1765/114 006 (1.5)

$1380 / 128682(1.1)$
$1.11(1.03-1.20)$

$1.02(0.95-1.09)$
Pediatrician

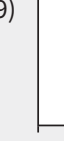

Adjusted OR $(95 \% \mathrm{Cl})$

Figure 1: Health outcomes of mothers, by type of primary care model, where the concordant model means mother and child were receiving care from the same family physician, the discordant model means mother and child were receiving care from different family physicians, and the pediatrician model means the mother was receiving care from a family physician and the child was receiving care from a pediatrician. $\mathrm{Note}$ : $\mathrm{Cl}=\mathrm{confidence} \mathrm{interval,}$ $\mathrm{ED}=$ emergency department, $\mathrm{OR}=$ odds ratio, ref. = reference.

concordant care may distract from adequate care for both the mother and the child. During any visit to their doctor, a mother may give her child's medical and emotional needs priority over her own. Physicians paid entirely, or largely, through fee-forservice (i.e., fee-for-service and noncapitated models) - about half of all primary care physicians in Ontario ${ }^{21,22}-$ may follow a
"1 problem per visit" policy. ${ }^{23,24}$ This may explain why we found a higher-than-expected number of maternal and child primary care visits, without a corresponding improvement in health system performance outcomes (e.g., low-acuity emergency department visits) among dyads that received concordant care. ${ }^{25}$ Second, a family physician delegated to care for just the woman 


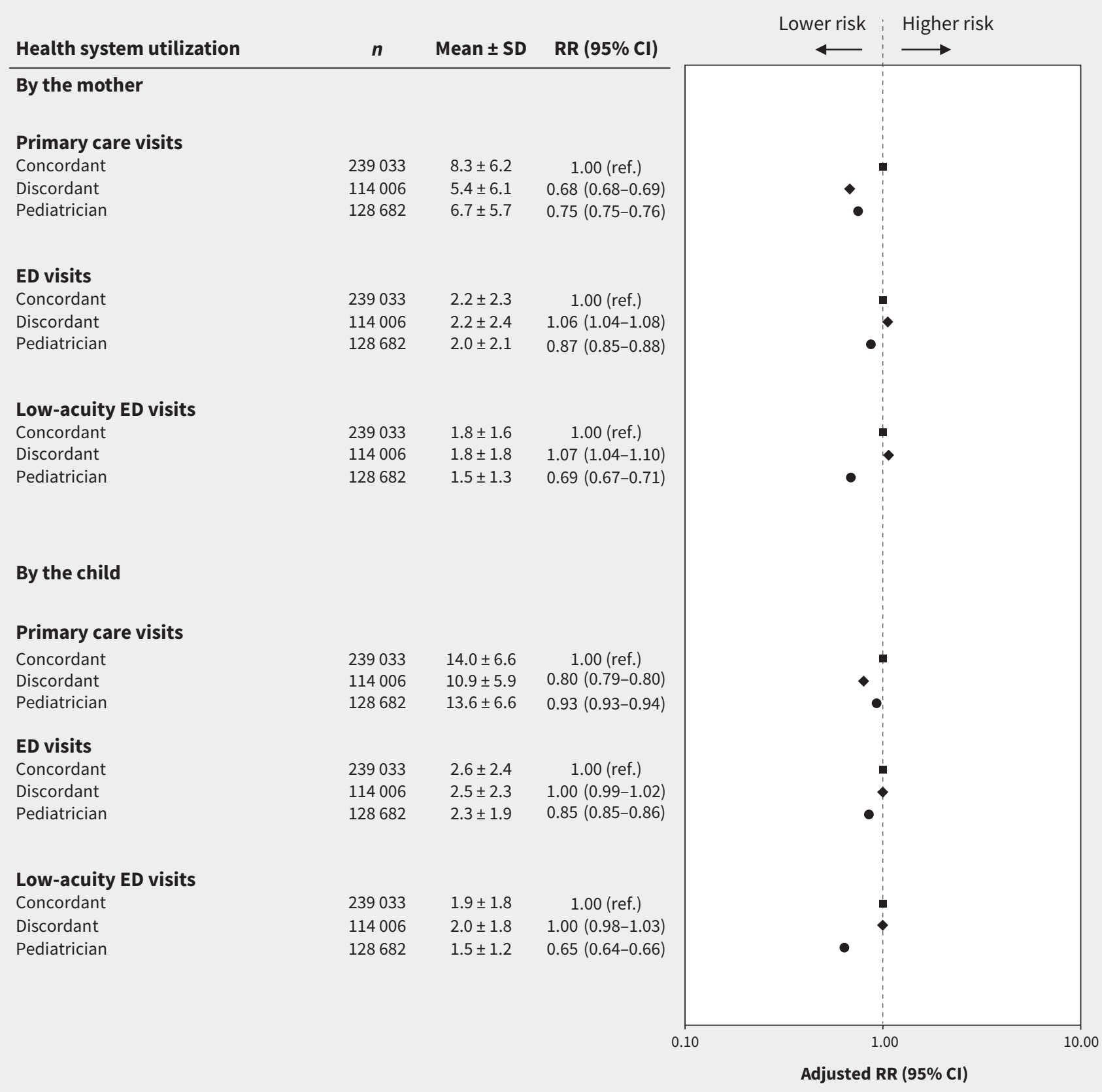

Figure 2: Health system utilization by mothers and their children, by type of primary care model, where the concordant model means mother and child were receiving care from the same family physician, the discordant model means mother and child were receiving care from different family physicians, and the pediatrician model means the mother was receiving care from a family physician and the child was receiving care from a pediatrician. Note: $\mathrm{Cl}=$ confidence interval, $\mathrm{ED}=$ emergency department, ref. = reference, $\mathrm{RR}=$ relative risk, $\mathrm{SD}=$ standard deviation.

may be better able to focus on her specific needs. Third, while pediatricians are trained to care specifically for children, their approach may be to include assessments of health, behaviours and relationships for the entire family. ${ }^{26}$ Pediatricians may recognize that to optimize the health of a child, the mother's own health needs must also be ensured, and they may therefore guide her to obtain support for herself. Thus, high-quality care from a child specialist may ultimately extend to mothers.
Fourth, although we adjusted for several important variables, such as neighbourhood income quintile and comorbidities, it is likely that residual confounding accounts for at least some of our findings. Specifically, unmeasured factors related to underlying child health risk may explain why we observed higher rates of child hospitalization in the pediatrician group. It is also possible that healthier mothers are more likely to enrol their infants in pediatrician-based primary care. 


\begin{tabular}{|c|c|c|c|c|}
\hline Outcomes for children & $n / N(\%)$ & $\begin{array}{l}\text { Adjusted OR } \\
(95 \% \mathrm{CI})\end{array}$ & Lower risk & $\stackrel{\text { Higher risk }}{\longrightarrow}$ \\
\hline \multicolumn{5}{|c|}{ Hospital admission (healthy children) } \\
\hline Concordant & $13724 / 218321(6.3)$ & 1.00 (ref.) & & \\
\hline Discordant & $5898 / 103629(5.7)$ & $0.92(0.89-0.96)$ & & \\
\hline Pediatrician & $7527 / 112577(6.7)$ & $1.14(1.10-1.18)$ & & b \\
\hline \multicolumn{5}{|c|}{ Hospital admission (chronic condition) } \\
\hline Concordant & 2682/20 $712(12.9)$ & 1.00 (ref.) & & \\
\hline Discordant & $1179 / 10377(11.4)$ & $0.86(0.79-0.94)$ & & \\
\hline Pediatrician & $2865 / 16105(17.8)$ & $1.41(1.32-1.50)$ & & $\bullet$ \\
\hline \multicolumn{5}{|l|}{ Death (healthy children) } \\
\hline Concordant & $72 / 218321(0.033)$ & 1.00 (ref.) & & \\
\hline Discordant & $45 / 103629(0.043)$ & $1.98(1.24-3.15)$ & & $\bullet$ \\
\hline Pediatrician & $32 / 112577(0.028)$ & $1.45(0.92-2.28)$ & & $\bullet$ \\
\hline \multicolumn{5}{|c|}{ Death (chronic condition) } \\
\hline Concordant & $39 / 20712(0.188)$ & 1.00 (ref.) & & \\
\hline Discordant & $37 / 10377(0.356)$ & $1.80(1.14-2.83)$ & & $\longrightarrow$ \\
\hline Pediatrician & $62 / 16105(0.385)$ & $1.72(1.14-2.60)$ & & $\bullet$ \\
\hline \multicolumn{5}{|c|}{ No 18-mo visit (healthy children) } \\
\hline Concordant & 40 070/96 719 (41.4) & 1.00 (ref.) & & \\
\hline Discordant & $25537 / 53066(48.1)$ & $1.06(1.03-1.09)$ & & \\
\hline Pediatrician & $13887 / 51077(27.2)$ & $0.47(0.46-0.49)$ & $\bullet$ & \\
\hline \multicolumn{5}{|c|}{ No 18-mo visit (chronic condition) } \\
\hline Concordant & 4072/9632 (42.3) & 1.00 (ref.) & & \\
\hline Discordant & $2851 / 5426(52.5)$ & $1.29(1.18-1.40)$ & & $\bullet$ \\
\hline \multirow[t]{2}{*}{ Pediatrician } & $2552 / 7990(31.9)$ & $0.61(0.56-0.65)$ & $\bullet$ & \\
\hline & & & & \\
\hline
\end{tabular}

Figure 3: Health outcomes of children, by type of primary care model and by the child's general health status, where the concordant model means mother and child were receiving care from the same family physician, the discordant model means mother and child were receiving care from different family physicians, and the pediatrician model means the mother was receiving care from a family physician and the child was receiving care from a pediatrician. Note: $\mathrm{Cl}=$ confidence interval, $\mathrm{OR}=$ odds ratio, ref. $=$ reference.

We found small but important differences in health and health system performance outcomes in mother-child dyads in relation to the nature of primary care delivery. For physicians who provide care to both a mother and her child, attention needs to focus on both patients. Leveraging of a clinician's knowledge of family dynamics and health and social needs could be optimized to improve health and system performance. Given that our study showed that more than a quarter of dyads involved care by a pediatrician, it is important that pediatricians continue to receive training that considers the whole family, even while focusing on the child. ${ }^{27}$ We speculate that such training to address family issues and offer support may partly account for our findings. Tenets of pediatriciandelivered primary care ${ }^{28}$ applied universally and more deliberately to existing family physician models of practice, could potentially improve outcomes for mother and child. For example, 
breastfeeding-friendly offices could be scaled up, and remuneration could be improved, to better value time spent addressing the needs of both mother and child. More nuanced shifts in training and care delivery could include a greater focus on child- and family-friendly practices in the medical home, as suggested by leading pediatric organizations: ${ }^{28,29}$ acknowledging the family as a constant in a child's life, building on family strengths, honouring cultural diversity and family traditions, recognizing the importance of community-based services, promoting an individual and developmental approach, and encouraging family-to-family peer support.

\section{Limitations}

We could not establish causation, nor were we able to determine whether the findings were related to other, unmeasured factors related to individual patient characteristics in different models of care or in how mothers selected their model of primary care. Physicians' characteristics and models of their practices were broadly included in the analysis in terms of remuneration and team-based care, but detailed information about practice structure, including access to allied health and practice-specific afterhours availability, was not available nor explored in this study; such factors may have contributed to our findings. We included almost the entire population of primiparous mothers in Ontario, but our findings may not be generalizable to multiparous women and their children. Although the study outcomes measured here can be mapped onto established primary care performance measurement indicators, and although they address a gap in our understanding of maternal and child primary care delivery, they are not comprehensive. Complementary to the current study would be the collection of survey data from parents and practitioners, and the determination of whether the current findings differ when primary care is provided by nurse practitioners or within a community health centre, settings that account for less than $1 \%$ of the Ontario population. ${ }^{21}$

\section{Conclusion}

We found that concordant care provided to a new mother and her infant by the same family physician did not, as we had expected, appear to confer better maternal or child health or health system performance outcomes than discordant family physician or pediatrician care. Further exploration of the specific aspects of pediatric primary care that may contribute to better maternal and child outcomes is warranted.

\section{References}

1. Rourke baby record. Ottawa: Canadian Paediatric Society; updated 2017 Nov. 17. Available: www.cps.ca/en/tools-outils/rourke-baby-record (accessed 2019 Jan. 24).

2. AAP schedule of well-child care visits. Itasca (IL): American Academy of Pediatrics; updated 2018 Oct. 26. Available: www.healthychildren.org/English/family -life/health-management/Pages/Well-Child-Care-A-Check-Up-for-Success.aspx (accessed 2019 Jan. 24).

3. Katz M, Rubino A, Collier J, et al. Demography of pediatric primary care in Europe: delivery of care and training. Pediatrics 2002;109:788-96.

4. Carter R, Riverin B, Levesque JF, et al. The impact of primary care reform on health system performance in Canada: a systematic review. BMC Health Serv Res 2016;16:324.
5. Macinko J, Starfield B, Shi L. The contribution of primary care systems to health outcomes within Organization for Economic Cooperation and Development (OECD) countries, 1970-1998. Health Serv Res 2003;38:831-65.

6. van Esso D, del Torso S, Hadjipanayis A, et al.; Primary-Secondary Working Group (PSWG) of European Academy of Paediatrics (EAP). Paediatric primary care in Europe: variation between countries. Arch Dis Child 2010;95:791-5.

7. Gill PJ, O'Neill B, Rose P, et al. Primary care quality indicators for children: measuring quality in UK general practice. Br J Gen Pract 2014;64:e752-7.

8. Ewald DA, Huss G, Auras S, et al. Development of a core set of quality indicators for paediatric primary care practices in Europe, COSI-PPC-EU. Eur J Pediatr 2018;177:921-33.

9. Huber CA, Brändle M, Rapold R, et al. A set of four simple performance measures reflecting adherence to guidelines predicts hospitalization: a claimsbased cohort study of patients with diabetes. Patient Prefer Adherence 2016; 10:223-31.

10. Blozik E, Reich O, Rapold R, et al.; Swiss Quality Indicator for Primary Care (SQIPRICA) Working Group. Evidence-based indicators for the measurement of quality of primary care using health insurance claims data in Switzerland: results of a pragmatic consensus process. BMC Health Serv Res 2018;18:743.

11. Davies SM, Geppert J, McClellan M, et al. Refinement of the HCUP quality indicators. Report No.: 01-0035. Rockville (MD): Agency for Healthcare Research and Quality (US); 2001.

12. Johnston S, Hogel M. A decade lost: primary healthcare performance reporting across Canada under the Action Plan for Health System Renewal. Healthc Policy 2016;11:95-110.

13. Guttmann A, Klein-Geltink J, Kopp A, et al. Uptake of the new fee code for Ontario's enhanced 18-month well baby visit: a preliminary evaluation. Toronto: Institute for Clinical Evaluative Sciences; 2011.

14. Emery DP, Milne T, Gilchrist CA, et al. The impact of primary care on emergency department presentation and hospital admission with pneumonia: a case-control study of preschool-aged children. NPJ Prim Care Respir Med 2015;25:14113.

15. A primary care performance measurement framework for Ontario: report of the Steering Committee for the Ontario Primary Care Performance Measurement initiative: phase one. Toronto: Health Quality Ontario; 2014.

16. Laberge M, Wodchis WP, Barnsley J, et al. Hospitalizations for ambulatory care sensitive conditions across primary care models in Ontario, Canada. Soc Sci Med 2017;181:24-33.

17. Data dictionary: data repository. Toronto: ICES. Available: https://datadictionary. ices.on.ca/Applications/DataDictionary/Default.aspx (accessed 2016 Apr. 11).

18. Chiu M, Lebenbaum M, Lam K, et al. Describing the linkages of the Immigration, Refugees and Citizenship Canada permanent resident data and vital statistics death registry to Ontario's administrative health database. BMC Med Inform Decis Mak 2016;16:135.

19. Williams J, Young W. Appendix 1: Summary of studies on the quality of health care administrative databases in Canada. In: Goel V, Williams JI, Anderson GM, et al., editors. Patterns of health care in Ontario. The ICES practice atlas. Ottawa: Canadian Medical Association; 1996:339-46.

20. McLeod L, Buckley G, Sweetman A. Ontario primary care models: a descriptive study. CMAJ Open 2016;4:E679-88.

21. Glazier R, Zagorski B, Rayner J. Comparison of primary care models in Ontario by demographics, case mix and emergency department use, 2008/09 to 2009/10. Toronto: Institute for Clinical Evaluative Sciences; 2012.

22. Saunders NR, Guan J, Fu L, et al. Periodic health visits by primary care practice model, a population-based study using health administrative data. BMC Fam Pract 2019;20:42.

23. Fullerton M. Understanding and improving on 1 problem per visit. CMAJ 2008;179:623.

24. Laberge M, Wodchis WP, Barnsley J, et al. Efficiency of Ontario primary care physicians across payment models: a stochastic frontier analysis. Health Econ Rev 2016;6:22.

25. Family medicine compensation and practice models in Ontario: compensation models. Toronto: Ministry of Health and Long-Term Care; updated 2019 July. Available: www.healthforceontario.ca/UserFiles/file/PracticeOntario/FM\%20 Compensation\%20Practice\%20Models\%20EN.pdf (accessed 2016 Jan. 15).

26. Schor EL; American Academy of Pediatrics Task Force on the Family. Family pediatrics: report of the Task Force on the Family. Pediatrics 2003;111:1541-71.

27. Zuckerman B. Two-generation pediatric care: a modest proposal. Pediatrics 2016;137:e20153447.

28. Committee on Hospital Care and Institute for Patient- and Family-centered Care. Patient- and family-centered care and the pediatrician's role. Pediatrics 2012;129:394-404.

29. Malouin RA. Positioning the family and patient at the center: a guide to family and patient partnerships in the medical home. Itasca (IL): American Academy of Pediatrics; 2015. 
Competing interests: Natasha Saunders received an honorarium as an Associate Editor for the Archives of Disease in Childhood and an honorarium for reviewing a grant from the MSI Foundation. No other competing interests were declared.

This article has been peer reviewed.

Affiliations: The Hospital for Sick Children (Saunders, Cohen); Department of Pediatrics (Saunders, Cohen), University of Toronto, Toronto, Ont.; ICES Central (Saunders, Ray, Diong, Guan, Cohen); Keenan Research Centre for Biomedical Science of the Li Ka Shing Knowledge Institute (Ray) and Department of Obstetrics and Gynecology (Ray), St. Michael's Hospital, Toronto, Ont.

Contributors: Natasha Saunders, Joel Ray and Eyal Cohen conceptualized and designed the study. Christina Diong and Jun Guan had access to and analyzed the data, and all of the authors contributed to interpretation of the results. Natasha Saunders drafted the initial manuscript. All of the authors revised the manuscript for important intellectual content, approved the final manuscript for publication and agreed to be accountable for all aspects of the work.

Funding: This study was supported by the Canadian Institutes for Health Research. The opinions, results and conclusions reported in this paper are those of the authors and are independent from the funding sources.

Data sharing: The data set from this study is held securely in coded form at ICES. Datasharing agreements prohibit ICES from making the data set publicly available, but access may be granted to those who meet prespecified criteria for confidential access, available at www.ices.on.ca/DAS. The full data set creation plan and underlying analytic code are available from the authors upon request, with the understanding that the programs may rely upon coding templates or macros that are unique to ICES.

Disclaimer: This study was supported by ICES, which is funded by an annual grant from the Ontario Ministry of Health and Long-Term Care (MOHLTC). The opinions, results and conclusions reported in this paper are those of the authors and are independent from the funding sources. No endorsement by ICES or the Ontario MOHLTC is intended or should be inferred. Parts of this material are based on data and information compiled and provided by the Canadian Institute for Health Information (ClHI) and Immigration, Refugees and Citizenship Canada (IRCC). The analyses, conclusions, opinions and statements expressed herein are those of the authors, and not necessarily those of $\mathrm{CIHI}$ or IRCC. Parts of this report are also based on Ontario Registrar General (ORG) information on deaths, the original source of which is Service Ontario. The views expressed are those of the authors and do not necessarily reflect those of ORG or the Ministry of Government Services.

Accepted: May 15, 2020

Correspondence to: Natasha Saunders, Natasha.saunders@sickkids.ca 